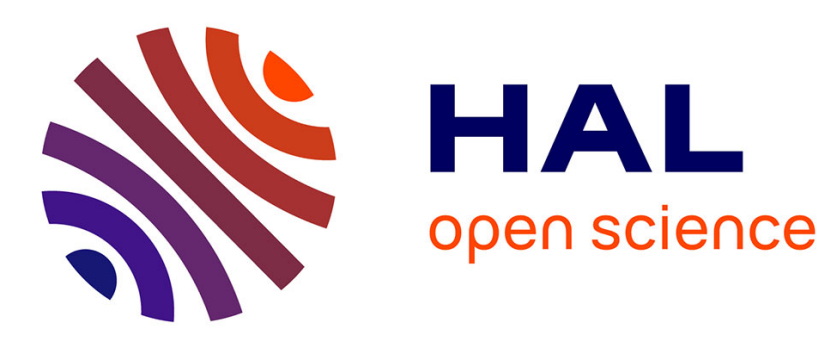

\title{
Histoire de la maladie mentale dans le Moyen-Orient médiéval
}

\author{
Ali Amad, P. Thomas
}

\section{To cite this version:}

Ali Amad, P. Thomas. Histoire de la maladie mentale dans le Moyen-Orient médiéval. Annales Médico-Psychologiques, Revue Psychiatrique, 2011, 169 (6), pp.373. 10.1016/j.amp.2010.06.023 . hal-00771587

\section{HAL Id: hal-00771587 https://hal.science/hal-00771587}

Submitted on 9 Jan 2013

HAL is a multi-disciplinary open access archive for the deposit and dissemination of scientific research documents, whether they are published or not. The documents may come from teaching and research institutions in France or abroad, or from public or private research centers.
L'archive ouverte pluridisciplinaire HAL, est destinée au dépôt et à la diffusion de documents scientifiques de niveau recherche, publiés ou non, émanant des établissements d'enseignement et de recherche français ou étrangers, des laboratoires publics ou privés. 


\section{Accepted Manuscript}

Title: Histoire de la maladie mentale dans le Moyen-Orient médiéval

Authors: A. Amad, P. Thomas

PII: $\quad$ S0003-4487(11)00064-3

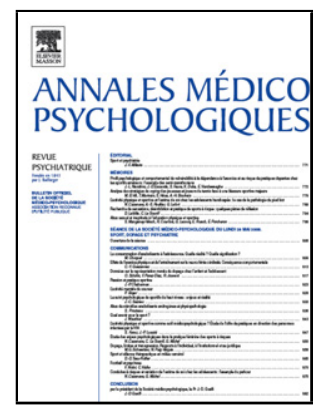

DOI: $\quad$ doi:10.1016/j.amp.2010.06.023

Reference: $\quad$ AMEPSY 1308

To appear in: $\quad$ Annales Médico-Psychologiques

Received date: $\quad 8-6-2010$

Accepted date: $\quad$ 27-6-2010

Please cite this article as: Amad A, Thomas P, Histoire de la maladie mentale dans le Moyen-Orient médiéval, Annales medio-psychologiques (2010), doi:10.1016/j.amp.2010.06.023

This is a PDF file of an unedited manuscript that has been accepted for publication. As a service to our customers we are providing this early version of the manuscript. The manuscript will undergo copyediting, typesetting, and review of the resulting proof before it is published in its final form. Please note that during the production process errors may be discovered which could affect the content, and all legal disclaimers that apply to the journal pertain. 


\section{Mémoire}

\section{Histoire de la maladie mentale dans le Moyen-Orient médiéval History of mental illness in Medieval Middle East \\ A. Amad ${ }^{*}$, P. Thomas \\ Service de Psychiatrie Adulte, Hôpital Michel Fontan, Centre Hospitalier Universitaire de Lille, France}

Auteur correspondant: A. Amad, Service de Psychiatrie Adulte, Hôpital Michel Fontan, Centre Hospitalier Universitaire de Lille, France

Adresse email : aliamad2@yahoo.fr

Texte reçu le 8 juin 2010 ; accepté le 6 septembre 2010

\section{Résumé}

La médecine arabe est le fruit de savoirs venant de plusieurs horizons; elle est notamment influencée par la médecine grecque. Les moines nestoriens ont joué un rôle capital dans le processus de transmission des connaissances du monde arabe au monde grec. Le savoir médical s'est développé et a été diffusé en parallèle de l'expansion de l'Islam au Moyen-Orient. En effet, la tradition musulmane a élevé la médecine au premier rang parmi les sciences. Ainsi, protéger et préserver la santé des individus est un principe religieux reconnu. Les troubles mentaux étaient considérés comme des maladies à part entière, et de très célèbres médecins de cette époque ont écrit et travaillé sur ce sujet. On distingue alors différents types de traitement comprenant des prises en charge psychothérapeutiques, pharmacologiques, musico-thérapeutiques et hospitalières dans les Bimaristans.

Mots clés : Bimaristan ; Contexte socio-économique ; Histoire ; Histoire médiévale ; Maladie mentale ; Médecine arabe

\footnotetext{
Abstract

Arabic medicine has multiple influences and Greek heritage is the most important. Nestorian monks played an essential role in transmitting knowledge. Medicine's development and diffusion coincided with Islam expansion. Indeed, Muslim tradition raised medicine in the first rank among sciences. Protect and preserve health of the individuals is a recognized religious principle. Mental disorders were considered as full-fledged diseases, and numerous
} 
illustrious physicians of this time wrote about this. Different kinds of treatment existed: psychotherapy, pharmacotherapy, music therapy and hospitalization in the Bimaristans.

Keywords: Arabic medicine; Bimaristan; History; Knowledge transmission; Medieval history; Mental illness; Treatment

« Où est donc cette vache que je la tue ? » demande Avicenne un couteau à la main, face à un prince délirant qui beugle et crie : «Je suis une vache ! Tuez-moi et faites un ragoût avec ma viande. » L'illustre médecin le palpe, déclare que la bête est trop maigre et indique qu'il faut l'engraisser. Pour se préparer au sacrifice, le malade accepte alors la nourriture qu'il refusait jusque-là. Ses forces reviennent et il guérit [9].

Voilà un exemple typique de traitement psychique utilisé dans la médecine arabomusulmane médiévale. Les médecins de l'époque disposent en effet d'un système de soin de la maladie mentale étonnamment moderne, comprenant des traitements médicamenteux, des soins psychothérapeutiques et des prises en charge hospitalières.

L'histoire de la maladie mentale dans le Moyen-Orient médiéval illustre parfaitement le lien essentiel entre un contexte socioculturel et économique favorable et l'émergence puis le développement d'une science.

Ainsi, l'essor de l'islam au VII siècle marque considérablement le Moyen-Orient. Les conquêtes des musulmans facilitent la transmission de connaissances médicales venant de plusieurs régions grâce aux traductions d'ouvrages en langue arabe et à l'assimilation de nombreuses cultures, conformément aux propos du prophète de l'Islam qui enseigne à ses compagnons d'aller «chercher le savoir jusqu'en Chine». Ce climat influence alors la perception des malades mentaux. Ces derniers sont peu stigmatisés, ils sont considérés comme des malades à part entière et la maladie fait partie du destin décidé par Dieu. Se soigner ou soigner son prochain étant une prescription religieuse, les hautes autorités sociales contribuent financièrement aux structures médicales, ces dons étant favorisés par la bonne santé économique de l'empire musulman jusqu'au XIII' siècle. D'ailleurs, le mot «majnoun» en arabe, qui signifie communément «fou », prend une connotation tantôt magique, tantôt scientifique, selon le contexte économique.

Les médecins arabo-musulmans développent alors des théories sur les maladies mentales et composent des traitements, encouragés par la prescription religieuse de soutien psychologique au malade. Les établissements tels que les bîmâristâns peuvent alors se 
développer, permettant de réelles prises en charge hospitalières. Le fonctionnement de ces hôpitaux a d'ailleurs probablement influencé Pinel lui-même, qui, dans son Traité médicophilosophique sur l'aliénation mentale, prenait en exemple, pour la prise en charge des malades mentaux, l'hôpital de Saragosse, fortement inspiré des bîmâristâns [2].

\section{Origines et influences de la médecine arabe}

Les origines de la médecine arabe sont par conséquent multiples. On distingue néanmoins une influence principale de la médecine grecque, elle-même dérivée de la médecine ancestrale ayurvédique fondée sur l'analogie entre le corps humain et le cosmos. Comment le savoir s'est transmis du monde grec au monde arabe ? La réponse à cette question apparaît avec l'étude des activités des moines nestoriens.

Ces moines sont originaires de l'empire byzantin. Ils sont chassés en 431, à la suite du concile d'Éphèse qui déclare leur doctrine hérétique. Ils choisissent alors de se réfugier dans l'empire perse et plus précisément en Mésopotamie. Leur rôle devient alors essentiel dans le processus de transmission des connaissances scientifiques. Les Nestoriens sont de culture grecque et possèdent de nombreux ouvrages de philosophie, de médecine, et de toutes sortes de sciences. La médecine qu'ils pratiquent est essentiellement hippocrato-galénique.

Les Nestoriens sont accueillis à Jondishapour (actuellement en Iran du Sud-Ouest), construite au III $^{\mathrm{e}}$ siècle par le roi Shapor $\mathrm{I}^{\mathrm{er}}$. Cette ville devient rapidement un centre intellectuel rayonnant, baignant dans les traditions zoroastrienne et perse, et comprenant un enseignement du grec et de l'indien. Son apogée est atteint au VII ${ }^{\mathrm{e}}$ siècle, durant lequel les néoplatoniciens d'Athènes sont accueillis et durant lequel la ville obtient le statut d'école de Jondishapour, véritable académie de médecine et de philosophie où l'enseignement tient une place primordiale. Elle devient le creuset de multiples cultures et de connaissances [12].

C'est ainsi qu'à Jondishapour se réunissent diverses influences, dont la médecine grecque avec sa théorie des humeurs, la tradition locale perse avec la pureté des corps, l'hygiène sexuelle et la pharmacopée, un certain apport copte et chinois, et enfin quelques connaissances indiennes (certains médecins étaient envoyés en Inde et revenaient avec des connaissances en pharmacopée, des instruments chirurgicaux...) [2].

En 638, Jondishapour est conquise par les musulmans. La langue arabe ainsi que l'Islam se répandent rapidement. Quant aux livres, ils sont pour la plupart traduits en arabe, notamment sous l'impulsion des califes abbassides (750-1258). D'ailleurs, la « Maison de la sagesse », Bayt al-Hikma, est créée sous Al-Ma'mun (IX siècle), il s'agit d'une bibliothèque 
et d'un institut de traduction, du grec et du perse vers l'arabe, ouvert aux intellectuels [5].

\subsection{Influence religieuse}

L’islam connaît un essor extraordinaire dès le début du viI ${ }^{\mathrm{e}}$ siècle. Les nombreuses conquêtes des musulmans font que les terres d'Islam se transforment rapidement en un vaste empire. En parallèle, la médecine se développe considérablement durant cette période. En effet, la tradition musulmane enseigne que la médecine est au premier rang parmi les sciences. Préserver et conserver la santé des individus est un principe religieux reconnu. D'ailleurs, le prophète de l'islam Muhammad (Mahomet) pratique la médecine. On raconte qu'elle lui aurait été enseignée par son ami Ibn Kalada, médecin célèbre formé à l'école de Jondishapour, à qui il envoyait les patients « compliqués » [6].

La médecine prophétique est surtout régie par les principes de la médecine des humeurs. Elle combat les croyances et les superstitions de l'époque, en luttant par exemple contre le port d'amulettes, les talismans, les prédictions astrologiques, la sorcellerie... De plus, elle distingue clairement les maladies du corps de celles de l'esprit, considérées comme des maladies à part entière [3]. On rapporte ainsi de nombreuses maximes prophétiques illustrant l'importance des moyens spirituels dans le traitement du malade. Il faut par exemple «faire preuve d'optimisme », ou « insuffler toujours de l'espoir au malade à qui l'on rend visite ». Cette médecine a une influence capitale. Plusieurs livres sont des recueils des principes médicaux prophétiques et font office de références pendant plusieurs siècles. On trouve d'ailleurs actuellement certains de ces préceptes dans les remèdes traditionnels de nombreux pays musulmans.

\section{Théories sur la folie}

\subsection{Leurs origines}

Dès l'Antiquité, Hippocrate ( $\mathrm{V}^{\mathrm{e}}$ siècle avant J.-C.) élimine les explications surnaturelles et donne un cadre médical aux troubles psychiques. Il distingue le phrénétis (trouble mental aigu avec fièvre), la manie (trouble mental aigu sans fièvre), la mélancolie (toutes sortes de troubles mentaux chroniques), l'épilepsie (comparable à la dénomination actuelle), l'hystérie (douleur, convulsion), et la maladie des Scythes (comparable au transvestisme) [10]. 
Galien (II $\mathrm{e}^{\mathrm{e}}$ siècle) lui succède et donne un nouveau statut aux maladies mentales en les qualifiant de somatiques. Elles résulteraient d'un déséquilibre des humeurs, d'où l'aphorisme « l'âme suit le corps ». Galien affine en outre la théorie des humeurs, ce qui permet à celle-ci d'être reprise par la médecine arabe qui va l'élargir et l'adapter.

Les Arabo-musulmans héritent alors de cette conception de la maladie mentale. L'influence religieuse permet aux médecins de développer une approche psychosomatique de la maladie mentale. Ils sont en effet guidés par des principes religieux utilisés et recommandés par le prophète tels que «insuffler l'espoir au malade », ou encore «la consolation spirituelle améliore la guérison ». Pour ces médecins, l'intrication des phénomènes physiques et psychiques devient un principe de base.

$\mathrm{Au} \mathrm{IX}{ }^{\mathrm{e}}$ siècle, Ishaq Ibn Omrane fait naître une position différente de la position orthodoxe de Galien. Pour lui, «l'âme suit le corps, mais l'inverse est également vrai ». Alors que Galien considérait que le soin de l'âme pouvait se faire uniquement à travers le corps, désormais on soigne l'âme par l'âme. C'est à cette occasion qu'apparaît un nouveau courant, que l'on peut qualifier de « traitement de choc». Celui-ci utilise les états émotionnels tels que la peur, la honte ou la colère pour contrebalancer les humeurs [9]. On rapporte d'ailleurs de nombreuses anecdotes souvent amusantes, faisant partie de la littérature de l'époque, illustrant ce type de traitement.

Dans l'une d'elles, un médecin traite une jeune patiente souffrant d'une impotence de l'épaule droite. L'examen de la jeune femme lui permet de comprendre que ce trouble a une origine psychique. Dès lors, le médecin invite cette patiente chez lui où il avait pris le soin de réunir auparavant une assemblée d'hommes. Une fois arrivée, la jeune femme voit le médecin s'approcher d'elle et lui arracher violemment son vêtement, de sorte que la patiente, prise d'un sentiment de honte, se sert de ses deux bras pour se couvrir...

\subsection{Médecins célèbres et théories}

La médecine, rappelons-le, tient l'un des premiers rangs parmi les sciences dans la religion musulmane. S'occuper de son prochain, le soigner, le soutenir psychologiquement sont autant de principes qui font partie intégrante de la pratique religieuse. Les plus grands médecins de l'époque, dont certains sont à l'origine d'ouvrages médicaux de référence étudiés jusqu'au XVIII ${ }^{\mathrm{e}}$ siècle, et ce jusqu'en Europe, sont alors naturellement inspirés de ces préceptes.

Ainsi, Ishaq Ibn Omrane, originaire de Bagdad au IX ${ }^{\mathrm{e}}$ siècle, est l'auteur du Traité sur la mélancolie, dont la description est étonnamment moderne [3]. La première partie du traité 
est donc consacrée à la présentation de la maladie. Elle est décrite comme une atteinte du corps qui a des méfaits sur l'esprit. Il explique que chez les patients mélancoliques, les idées tristes sont exagérées et erronées et ont des répercussions somatiques : une altération de l'état général, des insomnies, un amaigrissement, de la tristesse ou encore un affaiblissement des organes. Les facteurs déclenchants sont ensuite énumérés. Il peut tout aussi bien s'agir de souffrances de l'âme, de frayeur, de timidité que de perte d'amour ou de disparition d'un être cher. Plusieurs formes sont dénombrées : hypocondriaque, forme avec angoisse, formes délirantes ou encore hallucinatoires. La deuxième partie du Traité est réservée à la thérapeutique. Ibn Omrane expose le traitement «psychothérapeutique » qui consiste à consoler et à rassurer le patient, lui faire écouter de la bonne musique et lui conseiller des promenades à la campagne et dans les jardins fleuris. Les règles hygiéno-diététiques sont aussi exposées, avec des régimes adaptés à chaque malade (en lien avec le dérèglement humoral). Il indique la physiothérapie où les patients sont soignés par des bains, des exercices et des massages. Il expose enfin la chimiothérapie, qui explique comment administrer au souffrant des pastilles sédatives, des laxatifs ou des stimulants. Chaque médicament est, bien entendu, dosé et soigneusement administré aux heures prescrites. À aucun moment du traité ne sont évoquées des causes surnaturelles (possession, sorcellerie), pourtant prépondérantes à la même époque en Europe. De plus, on ne retrouve pas la notion de suicide, formellement interdit par l'islam : «Et ne vous tuez pas vous-mêmes. Dieu, en vérité, est Miséricordieux envers vous » (Coran, 4/29). Certains auteurs pensent que la cohésion sociale et religieuse de l'époque limitait ce risque ; on peut également penser que le tabou autour du suicide dépasse les époques et les cultures. C'est également ce même médecin qui a, entre autres, permis le développement de la médecine au Maghreb après sa venue à Kairouan [2].

Une autre figure de la médecine est Razès, médecin Perse du IX ${ }^{\mathrm{e}}$ siècle. Il tient la fonction de chef de l'hôpital de Bagdad [8] où il introduit le terme de «psychothérapie », qui signifie textuellement «traitement de l'âme ». Il a la réputation d'un grand psychosomaticien qui rédigea de nombreux écrits sur les troubles mentaux et sur la relation médecin/malade [2].

Ensuite, arrive l'incontournable Avicenne : médecin, philosophe et scientifique du $\mathrm{X}^{\mathrm{e}}$ XI ${ }^{\mathrm{e}}$ siècle. Il est l'auteur du célèbre Canon, qui est un répertoire de toutes les maladies ayant une influence phénoménale au Moyen-Orient et en Europe jusqu'au XVIII ${ }^{\mathrm{e}}$ siècle [13]. Le prince de la médecine y explique que les «remèdes psychiques doivent aider les médicaments », et que le « meilleur traitement consiste à renforcer la force psychique » [4]. Il décrit également une anorexie mentale chez un prince amoureux, illustrant l'importance des relations entre corps et esprit pour ces médecins [15]. 
Il faut mentionner également El Kindy et les Frères de la pureté, qui sont des maîtres en musicothérapie. Ils considèrent que la musique a une influence sur l'âme et l'esprit (concept grec d'ethos). Elle exprime 1'harmonie de l'ordre céleste. Les quatre cordes du luth sont liées au zodiaque, aux quatre éléments, aux quatre humeurs, aux quatre saisons, aux couleurs et aux parfums [3].

Enfin, Mohamed Essikily, «le Sicilien », est un médecin du XIII ${ }^{\mathrm{e}}$ siècle. Il fournit de nombreuses analyses sur le mouvement, le repos, ainsi que sur la transition « veille-sommeil». Il traite également les paralysies hystériques, ou au moins non organiques, par des décharges électriques de poissons torpilles, technique étonnante au regard des travaux concernant le traitement des conversions somatiques par stimulation magnétique transcrânienne.

Les plus grands noms de la médecine s'intéressent donc aux maladies mentales et préconisent l'accompagnement psychologique des patients. L'idée de causes psychiques aux maladies mentales a également été appuyée par le regroupement des malades mentaux et leur observation. Ibn Bakhtishu, médecin réputé du $\mathrm{XI}^{\mathrm{e}}$ siècle, dans son livre Message aux médecins, attire l'attention du lecteur sur les éléments psychiques négligés par les «médecins ordinaires » (qui ne sont pas entrés dans les bîmâristâns) [9].

\section{Les bîmâristâns}

\subsection{Origines}

Ce terme provient de deux mots perses : bimâr, qui signifie malade ; et stân, suffixe pour les noms de lieux. Au IV ${ }^{\mathrm{e}}$ siècle, dans l'empire byzantin, on trouve déjà ce que l'on appelle les xenodokeion, qui sont des institutions charitables chrétiennes organisées par les Nestoriens. Elles se présentent sous les formes d'hospices, d'hôpitaux et d'auberges, et sont construites dans toutes les grandes villes.

En Iran, à Nisibe au $\mathrm{V}^{\mathrm{e}}$ siècle puis à Jondishapour, les Nestoriens réfugiés créent des hôpitaux. Ceux-ci contiennent des lits pour les voyageurs, les malades et les indigents [11]. Avec l'avènement de l'Islam, on retrouve le premier hôpital en terre musulmane en 707, à Damas, qui porte désormais le nom de bîmâristân et qui innove. En effet, il est organisé en services, selon les pathologies traitées (chirurgie, ophtalmologie, médecine générale...), et on y trouve une section pour les malades mentaux. À la fin du VIII ${ }^{\mathrm{e}}$ siècle, le calife, successeur du prophète, demande aux médecins de l'école Jondishapour de créer un bîmâristân dans la capitale du monde musulman, Bagdad. Il s'agit là encore de rester conforme aux préceptes 
religieux de conservation et préservation de la santé des individus résumés par la parole prophétique: "Soignez-vous, car à chaque maladie correspond son remède, sauf la vieillesse. » Le modèle se propage alors à travers tout l'Empire.

\subsection{Fonctionnement}

On trouve des bîmâristâns fixes et ambulatoires, ces derniers étant nécessaires lors des mouvements de foule comme les guerres ou les pèlerinages. Les revenus sont assurés par les wakfs, qui sont des legs en argent, en nature, ou des héritages. Il s'agit d'institutions philanthropiques subventionnées par les califes, les sultans ou les rois.

Les bîmâristâns sont souvent construits au cœur de la ville, avec une architecture commune. Une cour rectangulaire avec un bassin est entourée d'un bâtiment central avec des ailes pour les cours, des magasins, une cuisine, une pharmacie et des latrines. Les bîmâristâns sont mixtes, mais les hommes et les femmes sont séparés, ainsi que des femmes médecins [14]. Les patients sont accueillis sans distinction d'origine ou de religion. Les médecins examinent les malades chaque matin, font préparer les remèdes et surveillent les régimes alimentaires. Ils assurent également un enseignement aux jeunes étudiants en médecine « au lit du malade ». Un diplôme de fin d'études permet de certifier les connaissances acquises. Les étudiants préparent aussi une thèse pour se spécialiser dans un domaine [16]. Les infirmiers sont qualifiés. Quant à la nourriture, elle est bonne et copieuse, et les locaux sont chauffés l'hiver [7].

\subsection{Soins apportés}

Les soins médicaux sont toujours apportés après l'examen du patient, avec l'aide d'ordonnances prescrites et envoyées à la pharmacie. Les remèdes à base d'une seule herbe sont toujours essayés avant les médicaments composés. Il existe des sédatifs comme l'opium et des stimulants. Par exemple, au $\mathrm{X}^{\mathrm{e}}$ siècle, Al Tamini invente un médicament appelé le « contentement de l'esprit», annoté de la façon suivante : «Clef pour la gaieté de l'âme et afin de se réjouir de toute peine. »

Les patients pratiquent aussi la gymnastique et doivent être massés. La musicothérapie occupe également une place importante dans le traitement. Des musiciens viennent régulièrement donner des spectacles auxquels ils font participer les patients. Des airs spécifiques sont composés selon le mal du patient (les luths ont quatre cordes, chacune 
représente une humeur). Le bassin creusé au milieu du bîmâristân a pour but de faire entendre le son du ruissellement de l'eau, ce qui calme les anxieux et les agités. Mais sa présence est aussi profondément liée à la médecine des humeurs, car l'eau du bassin humidifie l'air et lutte contre la sécheresse de la bile noire en excès chez les patients mélancoliques.

$\mathrm{Au}$ printemps, des fleurs sont dispersées par terre et leur odeur est censée monter directement au cerveau. Ainsi, les violettes froides et humides s'opposent aux qualités de la bile jaune. L'hôpital du Caire porte un grand intérêt à la qualité de l'air et à son parfum : il y existait une ingénieuse construction, qui, grâce à des éventails géants, faisait circuler l'air aromatisé avec certaines plantes. Il arrivait malheureusement que certains patients mangent les pétales des fleurs [9]...

\section{Conclusion}

Le survol de l'histoire de la maladie mentale dans le Moyen-Orient médiéval permet de montrer que le développement de la médecine est intimement lié au contexte culturel et spirituel de l'époque. Les préceptes religieux influencent en effet la conception de la médecine, et de la maladie mentale. Les moyens spirituels et le soutien psychologique sont recommandés par la religion musulmane pour faciliter la guérison. Cette optique, associée à la médecine des humeurs, influence considérablement la conception de la maladie mentale. De plus, soigner étant une recommandation religieuse, les rois, les califes soutiennent de façon matérielle la construction de bîmâristâns.

Il est alors intéressant de remarquer que la connotation du sens du mot arabe majnoun, signifiant communément «fou » évolue selon la période de développement économique. La racine arabe de ce mot renvoie à l'idée de ce qui est caché, dissimulé. Elle a d'ailleurs donné les mots signifiant folie (jounoun), esprit (jinn), fœtus (janin), ou encore paradis (janna). Ainsi, majnoun peut renvoyer au domaine de la pathologie mentale ou signifier «possédé par un jinn» (génie invisible qui peut être bon ou mauvais), alors qu'avant l'islam, le mot renvoyait au domaine de la magie et de la sorcellerie. Pendant l'âge d'or, le terme majnoun est largement connoté à la maladie mentale. Ainsi, l'immense majorité des cas sont considérés comme malade, la maladie faisant partie du destin décidé par Dieu. L'acceptation de ce destin a certainement permis de minimiser le sentiment de culpabilité chez les patients et le sentiment de honte de leur famille.

Après l'invasion de Bagdad par les Mongols au XIII ${ }^{\mathrm{e}}$ siècle, de nombreux remaniements socio-économiques ont lieu, entraînant une perte de cohésion sociale, la 
destruction de nombreuses institutions et la réduction des apports financiers de celles qui restent. L'organisation des soins change, les malades mentaux sont transférés dans des centres qui ressemblent à des centres de détention, induisant un isolement important et un support social moindre. La dimension de punition divine de la maladie mentale émerge et les patients deviennent responsables de leur état [1].

L'étude de cette histoire souligne alors l'importance du contexte socio-économique dans la stigmatisation de la maladie mentale. Elle doit nous faire réfléchir aux effets de la crise économique actuelle sur la perception de la maladie mentale dans la population générale, chez les médias et en politique. On ne peut alors que se demander à quel point le débat autour de la responsabilité des malades mentaux et l'ambiance sécuritaire autour de ces mêmes patients est lié aux difficultés économiques actuelles.

D'autre part, ce travail historique nous a conduit à une réflexion clinique transculturelle autour de la représentation de la maladie mentale (folie, dépression, suicide...), et des facteurs l'influençant chez les musulmans vivant dans des pays musulmans et chez les musulmans vivant en France. Les travaux dans ce domaine sont en effet souvent réalisés à partir de l'expérience d'un clinicien. Ainsi, un travail concernant ces interrogations est actuellement en cours à partir des résultats de l'enquête SMPG - «Santé mentale en Population générale : images et réalité »-du CCOMS. Cette approche nous permettra de discuter de façon plus objective pour savoir si la représentation de la maladie mentale diffère entre ces deux populations, et quels sont les éléments qui influencent ces différences, si elles existent.

\section{Conflits d'intérêt : aucun}

\section{Références}

[1] Al-Baldawi R. The interpretation of Madness "Jinon" in context of the socio-economic development of Islamic societies. WCPRR 2006. p.138-42.

[2] Ammar S. Histoire de la psychiatrie Maghrébine. In: Douki S, Moussaoui D, Kacha F. Manuel de psychiatrie du praticien maghrébin. Paris: Masson; 1987. http://www.vitaminedz.com/articlesfiche/0/305.pdf

[3] Ammar S. Médecins et médecine de l'Islam. Paris: éditions Tougui; 1984.

[4] Ammar S. Ibn Sina Avicenne : la vie \& l'œuvre. L’Or du temps; 2003.

[5] Balty-Guesdon M. Le Bayt al-hikma de Baghdad. Arabica 1992;39:131-50.

[6] Brewer H. Historical perspectives on health: Early Arabic medicine. The Journal of the 
Royal Society for the Promotion of Health 2004;124:184-7.

[7] Cloarec F. Bîmâristâns, lieux de folie et de sagesse : La folie et ses traitements dans les hôpitaux médiévaux au Moyen-Orient. Paris: L’Harmattan; 2000.

[8] Daghestani AN. al-Razi (Rhazes), 865-925. Am J Psychiatry 1997;154:1602.

[9] Dols MW. Majnūn: the madman in medieval Islamic society. Oxford: Clarendon press; 1992.

[10] First MB, Frances AJ, Pincus HA. DSM-IV-TR guidebook. Washington DC: American Psychiatric Pub; 2004.

[11] Le Coz R, Lazorthes G. Les médecins nestoriens au Moyen âge : les maîtres des Arabes. Paris: L'Harmattan; 2005.

[12] Miller AC. Jundi-Shapur, bimaristans, and the rise of academic medical centres. J R Soc Med 2006;99:615-7.

[13] Namazi MR. Avicenna, 980-1037. Am J Psychiatry 2001;158:1796.

[14] Pormann PE. Female patients and practitioners in medieval Islam. The Lancet 2009;373:1598-9.

[15] Shafii M. A Precedent for Modern Psychotherapeutic Techniques: One Thousand Years Ago. Am J Psychiatry 1972;128:1581-4.

[16] Syed I. Islamic Medicine: 1000 years ahead of its times. Journal for the International Society for the History of Islamic Medicine 2002;2:2-9. 